\title{
Corps social et constructions navales traditionnelles : de la technique aux symboles
}

Laura Bogani et Jacques Ivanoff

\section{(2) OpenEdition}

1 Journals

Édition électronique

URL : https://journals.openedition.org/tc/274

DOI : $10.4000 /$ tc. 274

ISSN : 1952-420X

Éditeur

Éditions de l'EHESS

\section{Édition imprimée}

Date de publication : 1 janvier 2001

Pagination : 1-18

ISSN : 0248-6016

\section{Référence électronique}

Laura Bogani et Jacques Ivanoff, « Corps social et constructions navales traditionnelles : de la technique aux symboles », Techniques \& Culture [En ligne], 35-36 | 2001, mis en ligne le 07 septembre 2012, consulté le 29 septembre 2022. URL : http://journals.openedition.org/tc/274 ; DOI : https:// doi.org/10.4000/tc.274

Ce document a été généré automatiquement le 29 septembre 2022.

Tous droits réservés 


\title{
Corps social et constructions navales traditionnelles : de la technique aux symboles
}

\author{
Laura Bogani et Jacques Ivanoff
}

1 Le bateau permet l'expression des rituels, développe l'esprit syncrétique des populations maritimes (et terriennes) et symbolise l'individu autant que le groupe social (village, ville, État). Il devient un symbole pour toutes les sociétés et la plupart des articles qui composent ce volume nous le montrent abondamment: symbole de l'être humain d'abord, il devient l'image d'un idéal social, puis un modèle religieux utilisé par les exégètes. Une expression accomplie de ce symbole se trouve dans le poème influencé par le soufisme du Monde malais que nous avons mis en exergue ${ }^{1}$. Cette représentation du bateau est souvent antérieure à l'arrivée de l'islam dans la péninsule, bien que certaines populations (les Sama décrits par A. Martenot) la développent avec l'arrivée d'un «chamanisme islamisé». Vladimir Braginsky (1998: 51), qui rapporte ces très beaux vers, remarque aussi que le symbole du bateau n'est qu'une composante d'un ensemble qui englobe d'une part l'arbre, l'oiseau, l'abri (maison ou sanctuaire), et d'autre part le soleil, la lune et le serpent. Nous voilà déjà au cœur des complexes mythologiques, des cosmogonies ou des thèmes des légendes de légitimation des royaumes asiatiques. Enfin, remarquons que le thème du bateau est très ancien, puisque dès la période dongsonnienne on en trouvait des traces, notamment sur les fameux tambours de bronze (M. Jacq-Hergoualc'h et P.-Y. Manguin).

2 Les articles présentés nous ont orientés vers l'océan Indien (L. Varadarajan, H. Kumar, S. Bhattatcharya, P.-Y. Manguin) - notamment ses bordures sud-est asiatiques (N. Hinshiranan, O. Ruohomäki, J. Ivanoff, L. Gansser) - mais aussi vers le Pacifique (C. Coiffier, I. Leblic, H. Guiot) et l'Asie du Sud-Est (Hoc Cheng Siny, M. Jacq-Hergoualc'h, V. Arnaud, J.-Cl. Amos, A. Martenot), sans oublier les bordures chinoises (E. Rieth). Ce volume s'inscrit dans une perspective de recherche nouvelle, bien que depuis des années des chercheurs, des marins, des architectes navals vouent leur intérêt aux bateaux, à leur devenir, à la construction traditionnelle, à la navigation, aux 
cérémonies et rituels. Ils travaillent pour comprendre et retrouver les bases d'une activité en voie de disparition. Les réserves de bois mal exploitées ne garantissent plus la matière première pour la réalisation des unités comme par le passé, malgré la persistance d'isolats, la Birmanie par exemple. C'est pourquoi l'intérêt porté aux pirogues monoxyles encore existantes est toujours justifié, car elles sont des jalons historiques permettant de comprendre l'évolution des techniques; et les auteurs les plus audacieux pourront définir des axes de recherche comparatifs et ethnoarchéologiques, confronter les traces épigraphiques qui recouvrent d'une manière troublante la forme des pirogues actuelles (M. Jacq-Hergoualc'h). La disparition - et/ ou la transformation -, à terme, de la plupart des bateaux traditionnels est inévitable, et il était nécessaire de présenter les données recueillies sur des terrains variés, données qui concernent les différents modes de construction navale ou l'emploi des bateaux comme moyen de transport, de pêche, de lieu de vie ou de cérémonie.

Le bateau dans l'aire Asie-Pacifique est présent dans maints domaines sociaux. Les populations installées le long d'un fleuve, d'un lac, en bord de mer, ou encore sur une île, vivent au rythme de l'eau; il faut rappeler ici que l'Asie du Sud-Est a toujours été une civilisation de l'eau, de l'eau douce en particulier mais sans que les deltas, et plus tardivement la mer, ne soient exclus. Ce qui explique peut-être l'omniprésence de la symbolique du crocodile (A. Martenot, C. Coiffier, etc.) à propos des monoxyles, d'abord embarcations fluviales. La frontière établie entre eau douce et eau salée (dans le monde chrétien et dans le Coran par exemple) représente géographiquement le delta, symboliquement la mise à l'épreuve de la foi et techniquement le passage du monoxyle de rivière aux embarcations de mer à bordés. Ce n'est qu'avec l'appropriation de la mer qu'une autre symbolique se développera, à caractère plus politique et " ésotérique ». La mer n'est pas un obstacle, elle ne sépare pas le continent des îles; elle constitue au contraire une liaison, une voie naturelle à parcourir afin d'entrer en contact avec d'autres civilisations ; de cette mission d'exploration, les mythes et les symboles ont su tirer certaines de leurs images. Chaque population a, selon ses besoins, son environnement, ses capacités, trouvé le juste rapport avec l'eau: faire flotter des traîneaux de bois pour les transporter en aval, utiliser des voiles pour partir au large, ramer dans une mare pour pêcher ou construire une pirogue à balancier pour aller plus vite. Cela s'accomplit toujours dans le respect de la tradition, des cérémonies et des rituels. Les génies, les esprits de l'eau, les ancêtres, comme les proues et poupes échancrées aux volutes élancées et aériennes, dont la forme s'inspire des bateaux des rois et plus largement des véhicules des dieux de l'Inde, tout cela s'inscrit dans une lignée religieuse, symbolique et rituelle donnant au groupe une forte marque identitaire dont la technique doit tenir compte.

\section{Revue historique et préhistorique}

4 Tous les auteurs « classiques » (J. Neyret, E. Pâris, J. Poujade, J. Hornell, etc.) ont réalisé des analyses historiques pour relier les bateaux d'aujourd'hui à des ancêtres communs, venus d'une sorte de «foyer initial » d'où la technique se serait diffusée. La quête des origines est légitime et nous entraîne dans les champs de l'archéologie (M. JacqHergoualc'h), de l'histoire (P.-Y. Manguin, E. Rieth) de l'ethnologie (N. Hinshiranan, I. Leblic, J. Ivanoff, C. Sather, A. Martenot), de l'architecture (C. Coiffier, L. Bogani), mais aussi de l'analyse des techniques (L. Varadarajan, H. Kumar, S. Bhattatcharya, P.-Y. 
Manguin). Les architectes autorisent la présentation de documents structurés de manière à pouvoir présenter les objectifs techniques des choix des hommes, leurs objectifs efficaces, leurs astreintes rituelles essentielles (O. Ruohomäki, J. Ivanoff, Hoc Cheng Siny) étant souvent mises "au placard». Il apparaît difficile de lier les deux domaines, technique et symbolique, ce qui montre que la technologie culturelle est loin d'être acceptée partout. La négation du symbolique au profit du «tangible» (pour reprendre le terme d'A. Martenot) n'empêche pas la présence de sculptures sur les embarcations, ni lents utilisations rituelles (cercueils par exemple) ${ }^{2}$. Ainsi, derrière les discours pragmatiques, le comportement des Sama révèle que sculpter un bateau, c'est changer sa fonction. Les choix rituels ou symboliques agissant sur l'efficacité technique (et pas toujours dans le sens d'une productivité accrue) ne sont pas des freins à l'innovation mais des ajustements structurels à des réalités auxquelles les groupes sociaux s'adaptent. Enfin, les marins (J.-Cl. Amos) et les amateurs éclairés (Etienne Sigaut présenté par E. Rieth) nous offrent une vision dynamique et une expérience personnelle qui nous exposent les résultats pratiques des choix techniques et symboliques des hommes.

\section{L'unité technique originale}

5 Certains auteurs anciens ont apprécié l'exotisme et la qualité des embarcations indigènes; ils ont voulu alors préserver et décrire ces œuvres, la «vision patrimoniale» dont parle E. Rieth à propos de l'amiral Pâris. Comme l'auteur le remarque si justement, l'ethnographie nautique est née au XIX siècle grâce aux travaux de ce fameux capitaine de corvette qui a su comprendre que les constructions traditionnelles révélaient des faits sociaux sans toutefois réussir à analyser ce lien dynamique et structurel entre les deux domaines. Certains auteurs classiques ont donc traversé les ombres de l'histoire et de l'ethnographie nautique, alors que d'autres tombaient dans l'oubli. Pourtant, les travaux de E. Sigaut mériteraient d'être sortis de cet oubli et $\mathrm{E}$. Rieth met bien en évidence ce qui le rapproche de Pâris, dont le succès est indéniable. Remarquons enfin à leur propos que, comme souvent en ethnologie au moins, ce sont les passionnés qui offrent les meilleurs travaux descriptifs et qui restent des sources inépuisables de références. Pourtant, s'ils ont su remarquer la faculté d'adaptation, ils n'ont pas compris qu'elle n'était que la conséquence d'un long combat entre tradition et modernité au sein des populations intéressées, et non pas un frein au «transfert technique » imposé par les Occidentaux; M. Charney (1997) a bien montré que la supériorité des bateaux de guerre (référence incontournable pour les historiens) était relative, notamment dans les deltas. Une innovation technique survenant dans un groupe de nomades (Sama, par exemple), de pêcheurs (Kanak, par exemple), ou dans un clan résulte d'un travail difficile auquel la coutume s'oppose. Mais cette sélection autorise cependant certains essais; seules les «bonnes idées » demeurent après que leur efficacité et leur supériorité ont fait leurs preuves, et pour autant qu'elles ne heurtent pas de front la coutume ; dans ce domaine, le pinisi indonésien (décrit par J.Cl. Amos) a remarquablement réussi. J. Neyret $(1974: 215)$ remarquait déjà cette faculté d'innovation.

«Ces pirogues démontrent un grand esprit d'initiative de la part de leurs constructeurs qui ont su repenser tous les détails de construction et introduire des solutions originales, non dépourvues de valeur. Ces pirogues sont en effet très pratiques, très manœuvrables et très rapides. » 
6 L'innovation et son succès -ou son échec- nous rappellent celle des pêcheurs de l'île des Pins en Nouvelle-Calédonie (I. Leblic) où, bien que souhaitée et peut-être nécessaire, elle se heurte à la tradition. Ce poids de la coutume n'autorise que certains essais techniques, reléguant les autres dans un oubli sans remède. Cette porte entrouverte sur le monde moderne permet toutefois, localement, l'expression originale des influences extérieures que subissent tous les peuples marins. L'itinéraire technique des peuples marins suit, dans une certaine mesure, les routes commerciales et religieuses ${ }^{3}$, ce qui n'empêche pas la survivance des traditions, ainsi des coques cousues de l'océan Indien. Ce n'est donc pas l'efficacité technique (bateaux de commerce pour longue distance par exemple) d'influence extérieure qui justifie l'innovation. Cependant, le fait que les grandes routes commerciales évitèrent longtemps le littoral birman explique ici la persistance de traditions séculaires. La connaissance de ces routes commerciales et donc la nécessité d'une perspective historique permet de mieux dessiner la "carte technique» qui divise notre aire: coque cousue/coque liée, gouvernail axial/gouvernail latéral, avec balancier/sans balancier...

7 I. Leblic décrit de façon pertinente le processus technique qui transforme un arbre en pirogue. Sa description peut s'appliquer à bon nombre d'autres populations décrites dans ce volume (Iatmul, Moken, Cambodgiens, Birmans, etc.). La connaissance empirique des cultures (le gabarit inscrit dans l'esprit du Tahitien dont parle H. Guiot ou la représentation du récif corallien en trois dimensions dont parle A. Martenot) s'est développée sur un terreau identique, répondant à des stimulations socio-économiques et écologiques équivalentes. Ce sont surtout les moyens de production qui ont fait la différence dans les méthodes de construction navale développées par la suite. D’une manière prononcée dans le Pacifique, chez les Kanaks ou les Tahitiens, l'acte efficace est un acte rituel. L'arbre doit être inspecté, écouté, palpé, ausculté; on chasse son esprit, on prépare sa chute. Nous voilà déjà dans le domaine des esprits de la forêt, des croyances dépassant le cadre de la construction technique. Mais l'Asie du Sud-Est, plus « symboliste " peut-être, n'est pas en reste. Les esprits «flottent » (Hoc Cheng Siny), poursuivent les hommes, montent sur le bateau (L. Gansser et J. Ivanoff). Cette phase d'abattage, primordiale pour définir l'avenir spirituel d'une embarcation, nous entraîne vers l'astrologie qui nous renvoie aux grands modèles indigènes et/ou indiens, que nous ne pouvons, malheureusement, pas développer ici.

\section{Comment évacuer le symbolique?}

8 Nos grands ancêtres, pionniers des descriptions des "pirogues indigènes » et autres "bateaux exotiques ", tiennent peu compte de la symbolique (réservée aux ethnologues par commodité) et ne font que mentionner l'aspect esthétique sans mettre en valeur son inscription dans un complexe culturel, le séparant ainsi du domaine technique.

«La coque est monoxyle avec éperon proéminent à l'avant: les bords sont surmontés de larges fargues découpées d'une manière fantaisiste à l'avant et à l'arrière à la manière de pièces de proue et de poupe ornementales. » (Neyret 1974 : 214).

Rien n'est fantaisiste et tout a une explication. Quoi qu'il en soit, J. Poujade pose clairement le problème de la position des chercheurs à l'époque. On cherche des routes, des migrations, des modes de diffusion, toute dynamique étant refusée aux populations « indigènes ». 
«Ces bateaux se rencontrent le long d'une des routes suivies par l'image du Bouddha ; elle traverse l'Inde du nord en suivant le Gange et aboutit en Birmanie ; deux voies s'en vont de là, l'une vers le centre de la Chine, l'autre aboutit à Angkor, puis paraît se diffuser en Malaisie. Tout au long des routes nous voyons des barques aux formes allongées ayant une vague silhouette de monstres marins, poissons, dragons et serpents; elles ont entre elles plusieurs éléments communs : la partie centrale de la coque a une forme en $\mathrm{U}$, la partie arrière en $\mathrm{V}$, autrement dit, les fonds sont arrondis sur tout le corps de ces bateaux qui sont généralement des pirogues creusées dans des troncs d'arbres, tandis que l'arrière a le fond aigu. L'avant est bas et l'arrière relevé comme la queue d'un poisson. La partie la plus large de la coque est généralement en avant du milieu. Tout cela rappelle bien la forme générale des bateaux longs de la Grèce classique. » (Poujade 1946 ; 275).

10 Aucun auteur n'a jamais ignoré la beauté des pirogues, l'habileté des charpentiers de marine, l'efficacité des mesures approximatives ou l'élégance des tontures par exemple. Aucun ne rejette l'aspect esthétique. Mais simplement, celui-ci ne peut, aux yeux de la plupart, être originel ; il doit venir d'un grand centre de diffusion : oiseau d'influence indienne contre crocodile d'origine déclarée indigène, sculptures géométriques d'origine indigène contre bouquet floral d'influence indienne, symboles religieux des barques royales d'origine indienne contre simples dessins indigènes, ces classifications sous-entendant une hiérarchie symbolique. Pourtant les anciens auteurs, amiraux, capitaines, architectes, missionnaires ont bien pressenti que les décorations avaient une dimension autre qu'esthétique. Tous ont laissé le soin de décoder ces symboles aux ethnologues, qui ont, pour leur part, coupé l'analyse symbolique de l'environnement technique. Les pirogues de Madura sont un bon exemple car elles avaient frappé J. Neyret, J. Hornell et l'amiral E. Pâris. Mais par exemple, la question des proues bifides, ou échancrées, n'a pas été résolue et ce volume y apporte quelques lumières. On sait que d'une manière générale, en Asie du Sud-Est, la proue élancée et affinée permet à une personne de se mettre à l'avant de l'embarcation pour pêcher ou pour assurer le passage dans les rapides. Son poids fait déjauger le reste du monoxyle. Il ne s'agit pas seulement de fendre la mer : un monoxyle, solide, à la proue droite permet aussi une meilleure pénétration dans l'eau. Une proue aplatie et effilée d'un monoxyle et une proue droite taillée dans le bloc monoxyle ont donc la même fonction : fendre l'eau et déjauger. L'explication technique de l'origine des proues échancrées n'est donc pas suffisante. D'un point de vue pratique, les échancrures permettent aussi de donner aux plongeurs des marchepieds, aux guerriers des éperons. Mais en allant plus loin encore, la forme accomplie des échancrures a aussi une fonction symbolique éminente, précédant son utilisation pratique (J. Ivanoff).

11 On traite de la symbolique sociale ou politique (bateaux austronésiens), de l'esthétique (pirogue polynésienne), du rituel (bateau/cercueil) puis de la technique en ne parvenant que rarement à lier celle-ci aux autres domaines. Le fait que les travaux d'ethnologie maritime puisent leur source dans ceux des marins et des architectes navals a considérablement influencé le domaine de la recherche sur la technologie navale. Ainsi, on traite bien des données techniques mais on laisse le social de côté comme si celui-ci n'était pas descriptible. Les architectes navals et les ethnologues n'ont pas les mêmes objectifs. Mais leur travail est complémentaire et devrait s'effectuer en commun puisqu'une telle "raison" technique ne peut parfois se comprendre qu'au regard d'une explication symbolique ou rituelle.

12 Plusieurs auteurs approchent la société de marins qu'ils connaissent à travers la technique, parce qu'ils sont eux-mêmes marins, ou parce qu'ils n'ont pas toujours le 
temps de considérer la société dans son ensemble (J.-Cl. Amos, P.-Y. Manguin, L. Varadarajan). D'autres envisagent le domaine de la construction navale à travers son seul aspect rituel (O. Ruohomäki), celui-ci étant assez général et classique (offrande, esprit de l'étrave, esprit des arbres). D'autres articles lient davantage les rituels à la construction (H. Guiot par exemple) et d'autres enfin s'attaquent aux relations entre le technique et le symbolique (P.-Y. Manguin et J. Ivanoff par exemple). Les rituels nous intéressent au plus haut point et révèlent des domaines insoupçonnés par l'analyse technique car ils mettent les chercheurs sur la voie de la compréhension du rapport entre efficacité technique et efficacité symbolique (et non plus sociale). Certains autres peuvent faire le lien entre technique et rituel (C. Sather, L. Bogani). On s'aperçoit à la lecture des articles que beaucoup d'auteurs (H. Guiot, P.-Y. Manguin, J. Ivanoff, A. Martenot par exemple) envisagent la construction navale circonscrite à une époque, une population ou à un type d'embarcation, tout en prenant en considération le contexte social, nécessaire pour comprendre pourquoi tous les peuples marins et toutes les grandes thalassocraties sont devenues des empires par un développement harmonieux et consensuel, des domaines de la technique et du symbolique; c'est pourquoi les analyses de P.-Y. Manguin, au niveau politique et globalisant, et celles de J. Ivanoff et A. Martenot, au niveau local et ethnique, sont des révélateurs de ce lien social qui se fait jour grâce à l'étude du bateau et de sa construction, tant technique que symbolique, étroite relation qui fait la force d'adaptation, de conquête et de survie des populations maritimes.

\section{Du rituel au politique}

Le bateau monoxyle, d'abord pirogue rituelle, devient d'usage commun; il s'affine, assume une fonction centrale dans le développement de la mobilité et de l'économie villageoise et devient le prolongement nécessaire des cités lacustres et des villages littoraux. Ce prolongement lui donne un rôle pivot : issu de la forêt primaire, le bateau incarne le premier élément séparant l'homme de son unité mythique (c'est-à-dire les règnes végétaux, animaux et minéraux). On lui donne vie ( $\mathrm{V}$. Arnaud) en séparant magiquement l'humain de son indifférenciation mythique, mais on l'intègre dans la famille (Cl. Sather) ou dans le clan (C. Coiffier). Abandonnant leur matrice primaire sylvestre, les esprits qui suivent l'arbre abattu investissent l'embarcation que les hommes avaient déjà assimilée, par leur travail communautaire et par leur association de compétences, à un rite social. Cette infiltration, cette deuxième vie, cette insufflation, quel que soit le nom qu'on lui donne, est accomplie d'une manière spécifique à chaque population, mais toutes conservent certains traits, ainsi des offrandes faites avant le lancement et les prières effectuées à la «tête » du bateau. Le monoxyle est devenu un double du corps humain, un rappel des mythes fondateurs, un prolongement technique d'une nécessaire identité culturelle. Il devient alors moyen de reconnaissance ethnique. Rappelons que les cérémonies sont également présentes, par exemple, dans le motif du bateau puisque, comme le remarque P.-Y. Manguin, le terme désignant le chamane du monde malais, pawang, ressemble beaucoup au terme désignant le « maître du bateau », puhawang.

En Asie du Sud-Est, le commerce se développe, notamment grâce au Malay network, mais aussi au commerce Inde-Chine et à toutes les variantes commerciales qui ont façonné l'Asie du Sud-Est et ses périphéries indiennes et océaniennes (routes trans-isthmiennes, 
détroit de la Sonde, désir de conquérir la Chine à travers l'Asie du Sud-Est à l'époque coloniale, etc.). Le bateau subit le contrecoup du développement, se transforme, d'où l'apparition de deux catégories. D'une part le bateau de transport s'agrandit avec les échanges et prend sa forme accomplie dans le bâtiment de guerre sculpté par exemple sur les bas-reliefs du Bayon et que nous présente M. Jacq-Hergoualc'h. Il devient une réalisation sociale, un effort conjugué des membres d'une société hiérarchisée qu'il reflète, un symbolisme social puisant sa force dans la construction communautaire d'une pirogue monoxyle (H. Guiot); il en devient le symbole (P.-Y. Manguin). Mais d'autre part, ce développement social se réalise d'abord à partir des monoxyles et des premiers bordés, quand le bateau n'est encore qu'un prolongement de l'homme et l'image d'une population s'appropriant son environnement. Nous pouvons donc identifier trois stades dans le développement d'une technologie symbolique : humain, rituel (et spirituel) sociopolitique.

\section{Bateau et corps humain}

La plupart des auteurs rappellent l'homologie des termes qui désignent respectivement les parties du corps humain et celles du bateau (J. Ivanoff, C. Coiffier, Cl. Sather), notamment du monoxyle. Cette identité entre la coque et le corps humain puise ses origines dans l'analogie entre l'homme et les végétaux, qui renvoie à l'unité mythique primordiale et à la sortie de l'arbre de sa matrice sylvestre. Le bateau unit donc la terre, la mer et l'homme. Il ne s'agit en aucun cas de briser l'unité mythique et le bateau devient alors une extension de l'homme intégré dans un environnement maîtrisé à l'aide d'un appareil rituel, symbolique et religieux. Le bateau permet aux hommes de retrouver le sens de leur mythe. On retrouve ce désir d'unité, notamment chez les nomades marins décrits par CI. Sather, A. Martenot, J. Ivanoff, N. Hinshiranan: l'organisation à bord reflète une organisation cosmologique. Chaque chose a sa place et déranger cet ordre a des conséquences sur le groupe et ses individus (naissance difficile, séparation du couple, chavirage, etc.). Cette idée existe chez nos marins contemporains, pour qui l'organisation est une préoccupation nécessaire à la bonne marche d'un bateau. Elle a bien sûr perdu ses relations avec le macrocosme.

\section{Rituel et spirituel}

Sur chaque embarcation vivent des esprits, génies, ancêtres ou dieux. Ils viennent de la forêt ou de la maison, prolongeant alors un culte domestique. Issus de traditions religieuses anciennes, ils viennent des eaux douces et s'adaptent à la mer. Les embarcations monoxyles ont de tout temps été comparées à des animaux, des esprits et des dieux ; leurs formes et leurs lignes s'inspirent de celles des oiseaux, mais aussi de celles des dragons (pour les pirogues rituelles et royales). J. Neyret remarquait déjà que les embarcations préhistoriques de l'Europe septentrionale (par exemple les sculptures de la roche Bohulsan datant de l'âge du fer) avaient des quilles prolongées aux deux extrémités, à l'avant par une sorte d'éperon relevé, à l'arrière par un talon incliné. Il note :

«Les formes de l'avant et de l'arrière sont très relevées: sur le type 1, la proue se relève et se termine en forme de monstre ; la poupe, également relevée, représente sans doute la queue. Sur le type 2, les deux extrémités sont contournées vers 
l'intérieur en forme de volute. Les extrémités [du type 3, plus récent] sont très relevées et identiques, mais les prolongements de la quille à l'avant et à l'arrière ont disparu. » (Neyret 1974, tome II : 296). asiatiques et océaniens. Elle orne les poteaux aux esprits moklen, les temples funéraires khmers, etc. L'oiseau est le modèle choisi pour désigner les lignes d'un bateau et il rappelle les montures ailées et divines venues de l'Inde. Il est en outre un animal psychopompe et le bateau lui-même fournit aussi un moyen de pénétrer le monde de l'au-delà, d'y amener les âmes et d'en ramener les hommes transformés. Les dessins, sculptures et autres frises sur bois sont le plus souvent situés à la proue et à la poupe ; çà et là, la coque elle-même est peinte et décorée, ce qui est plus fréquent dans le monde océanien mais parfois aussi en Indonésie (grande pirogue à balancier double de Lombok).

Ancêtres, dieux, esprits ou génies sont présents dans les embarcations et reflètent un état social et technique, rappelant l'ordre cosmologique dont nous venons de parler. Les embarcations royales et rituelles se rapportent aux dieux, alors que pour les embarcations du « commun » ne subsistent que des esprits avec des traces historiques d'ancêtres plus ou moins divinisés, comme Adam et Ève dont la présence évanescente hante les bateaux des Moken sans que ceux-ci en soient toujours conscients. Les ancêtres côtoient donc les esprits. Parfois assimilés aux trois ancêtres des bateaux moken (Ivanoff 1999), ils rappellent les trois déesses invoquées par les Cambodgiens la "Grande Dame Haira », la "Grande Dame Noire ", la demoiselle "Kantong bleu » (Hoc Cheng Siny). Ces trois personnages représentent l'eau, la terre et les arbres. Mais ils cohabitent ou parfois deviennent des esprits, avatars de divinités plus anciennes. Le bateau est l'espace du syncrétisme technique, social et religieux.

L'étrave est le plus souvent le lieu de résidence des esprits; elle reçoit les offrandes et constitue sur tous les bateaux le lieu intouchable et sacré. D'où le problème soulevé par o. Ruohomäki à propos des touristes qui s'allongent sur l'étrave. Si la survie des ethnies, ou groupes sociaux, passe par le tourisme, celui-ci vide la construction de son âme. C'est la porte ouverte à l'adaptation ou à l'acculturation ${ }^{4}$. C'est là que se situe son âme " officielle", mais plusieurs populations (Birmans, Moken, Iatmul, par exemple) dissimulent mal d'autres esprits plus complexes cachés dans la structure même du bateau. Ainsi le rituel et le symbolique sont-ils susceptibles d'expliquer les classifications locales des embarcations. Les esprits peuvent également être dispersés dans le bateau aux points stratégiques sélectionnés par la tradition et les innovations. À l'image des Thaïlandais qui réalisent trois emplacements destinés à recevoir des offrandes d'or, d'argent et cuivre, les navigateurs moken percent parfois trois trous (comme les mortaises des pinisi décrits par J.-Cl. Amos) dans le «nez» de l'étrave (ainsi que la nomment les Vietnamiens ou les Tahitiens, par exemple). Cela rappelle le calfatage d'or décrit par V. Arnaud chez les Yami de Botel Tobago, où le bateau reçoit, entre autres, des offrandes d'or destinées à assurer sa prospérité et surtout à l'assimiler à une femme. Ce qui nous ramène au rôle des femmes; rôle rituel et symbolique concernant la fécondité mais aussi permettant une classification sexuelle essentielle (O. Ruohomäki) quant aux pièces du bateau, au bateau lui-même, ou enfin, son esprit, son 
âme, son identité. Les trois endroits secrets, cachés dans l'étrave et destinés à accueillir trois offrandes, rappellent aussi les trois faces des pirogues royales thaillandaises, vietnamiennes ou cambodgiennes (L. Bogani, Hoc Cheng Siny) et, peut-être aussi, les Trois Joyaux du bouddhisme. Ce syncrétisme permet de comprendre les différents niveaux de croyance d'un groupe et aussi de découvrir, par exemple, les qualités associées aux jours, fastes et néfastes, aux couleurs, rouge et jaune, couleurs dominantes pour les tissus offerts aux esprits et enroulés autour de l'étrave, etc., bien que l'influence de l'islam tende à supprimer le rouge, par exemple. Toutes les cérémonies ne sont pas spécifiques aux bateaux, car elles comprennent souvent les offrandes communes à tous les bouddhistes et à tous les cultes régionaux de l'Asie du Sud-Est dans son extension indienne et pacifique (encens, bétel, bougie, fleurs, eau lustrale, etc.).

\section{Le bateau considéré comme animal marin fabuleux}

21 Le bateau est le véhicule des dieux, du Bouddha, du roi ; il symbolise le passage de la vie, la lutte contre les passions... L'association entre les échancrures des proues (ou proues bifides) et les symboles est connue depuis longtemps. J. Neyret en parlait déjà à propos des pirogues monoxyles de Lombok et de Bali.

«Ces pirogues monoxyles [de Lombok] sont de petites dimensions et de forme élégante. La proue est "bifide", technique que nous avons déjà rencontrée aux Philippines, dans les îles Sulu ainsi qu'à Minahasa (nord des Célèbes) et que nous retrouverons à Java. Une fourche est fixée à lavant pour recevoir les agrès. Elle [la pirogue de Bali] est souvent sculptée en forme d'oiseau. [...] La coque de ces pirogues [possède de] petites érections ornementales fixées sur les extrémités [...]. Elles sont à motif floral, en sculpture ajourée.» (Neyret 1974 : 210-212).

Ces proues bifides de pirogues monoxyles annoncent déjà le nomadisme futur. Elles avaient pour but techniquement avouable d'allonger la pirogue, de faciliter sa pénétration en mer, de servir de marchepied et de résister aux vagues. Plus les groupes s'éloignaient et prenaient possession de leurs embarcations comme d'une maison, plus les proues s'évasaient et s'ornaient de symboles.

Les différents visages que revêt l'image du bateau permettent une circulation et symbolisent des passages, des transitions, des évolutions possibles dans le temps et les espaces. Le bateau, avatar d'un animal fabuleux est alors une embarcation symbolique vers un au-delà. Il devient psychopompe et même un candidat à l'initiation. On en trouve des exemples particuliers avec les bateaux des héros de l'Insulinde (cf. A. Martenot et P.-Y. Manguin), mais aussi, avec ceux, plus proches de nous, de Jason ou d'Ulysse. Le bateau de Jason est magique et celui d'Ulysse lui permet de franchir des espaces où se manifestent les créatures marines fabuleuses. Le vaisseau mène le héros au combat contre les monstres (Thésée va combattre le Minotaure). Il n'est donc pas le lieu maléfique décrit par certains historiens aux visions eurocentristes ne voyant dans le bateau qu'un moyen de commerce, de guerre et d'exploitation d'une main-d'œuvre considérée comme insignifiante. Il est toujours question de combat contre les forces du mal, les passions, et de récompenses : la femme et le pouvoir. Dans les poèmes des navires d'origine indonésienne, le passage de l'homme sur terre est comparé à une traversée maritime, un symbole quasi universel qui a permis aux grandes religions d'imposer certains de leurs préceptes moraux. 

l'immortalité ; ainsi de Gilgamesh, le héros de l'épopée mésopotamienne, qui traverse les eaux de la mort et échoue à « cueillir » la plante d'immortalité au fond des mers. La mer est le lieu de la quête de l'immortalité et du Graal, du combat contre les passions (les monstres) et de la transformation. C'est pourquoi nombre de mythes rapportent l'histoire d'un corps démembré lié à l'élément liquide: Osiris en Égypte ancienne, Dionysos et Orphée en Grèce antique, Romulus à Rome, Lemminken en Finlande, Tampug chez les Palawan..., toutes figures mythologiques liées à la croissance et à la renaissance, par conséquent à la végétation (comme Osiris), ce qui rapproche du thème de l'enfant sacrifié et démembré à l'origine de l'âme du riz en Asie. Ces mythes liés à la fécondité existent encore chez les peuples de marins, les fameuses courses de pirogues et autres régates ( $c f$. A. Martenot) étant des avatars des rites terrestres liés à la culture du riz et célébrant la mousson.

Dans les épopées, les embarcations utilisées sont de ce fait des passeurs pour les héros ; ce sont aussi des contenants (arche, panier où l'on met les bébés héroïques abandonnés), c'est-à-dire le symbole du ventre maternel (rappelons qu'en Cambodgien, accoucher c'est aussi traverser les eaux); cela nous ramène au thème de l'enfant abandonné dans un panier ou un coffre, symbole de sa victoire sur la mort et de sa capacité à accomplir son destin, ce que G. Bachelard (1976) rappelle en voyant dans l'abandon de l'enfant sur les flots une terre qui se débarrasse de ce qu'elle ne peut accepter ; l'exclu de la terre retourne dans son élément de mort : la mer. Il s'agit donc de vaincre la mort, c'est-à-dire soit d'un processus d'individuation, thème cher à C. G. Jung (1964, 1932), soit de l'entrée définitive dans les Terres Pures, les îles des Bienheureux ou dans le royaume où réside l'Imam caché (Christinger et al. $1980: 22$ ). La littérature celtique rapporte elle aussi de nombreuses errances maritimes ; les héros ne sont plus maîtres de leurs embarcations et se laissent porter au gré des flots et des vents (navigation de Maelduin, navigation des Hui Corra, navigation de Snegdus et MacRiagla).

Ce rôle de moyen de transport symbolique du bateau va être repris par les modèles religieux dominants de l'Asie. Par exemple, le naga devint un protecteur du bouddhisme, et de « monstrueux », le voilà devenu vertueux, sa puissance s'en trouvant accrue; le voilà même érigé en modèle pour les embarcations de courses dans toute l'Asie, assurant la succession des pirogues en formes de dragons en Chine ou de serpent en Inde. Il devient alors le bateau-support du bouddhisme, comme il supportait Vishnu entre deux créations du cosmos. Il est donc comme les bateaux des mythologies égyptienne, nordique, grecque..., un véhicule symbolique de la mort et de la renaissance. Le monstre et notamment le serpent -on en trouve des traces dans le Livre des Morts égyptiens, sur les stèles funéraires vikings de l'île de Gotland- sont parfois assimilés au bateau lui-même, car embarcation et monstre sont les vecteurs de l'accomplissement de la destinée humaine, l'image de la plénitude du wagan des Iatmul, une sorte d'ouroboros, comme le remarque C. Coiffier. Les volutes du fleuve Sépik associées au serpent sont à l'image du Delta du Mékong assimilé à neufs dragons.

Les rois de Birmanie possédaient un bateau revêtu d'écailles de naga. Nous pensons qu'il symbolisait la diffusion du bouddhisme et le voyage de l'homme à travers ses réincarnations. Les embarcations de Patani, dont les coques sont des florilèges de figures monstrueuses issues de la forêt Himaphan sont un lointain souvenir de cette tradition. De fait, tous les bateaux de l'Asie du Sud-Est font écho à cette tradition 
religieuse qui prend pour symbole les formes concrètes du bateau, image du hamsa, véhicule de Brahma, ou bien du naga ou garuda, véhicule de Vishnu ou du makara dont les défenses peuvent devenir des éperons (M. Jacq-Hergoualc'h), ce dernier animal fantastique crachant lui-même parfois un naga symbolisant le cycle des renaissances. Cette imagerie $\mathrm{du}$ bateau comme support de la religion et véhicule de l'accomplissement et du dépassement de soi se retrouve chez les chrétiens. En effet les églises -les nefs- ne sont-elles pas construites sur des modèles de bateaux ? Le bateau, et/ou le monstre qui lui est associé, sont par conséquent les représentants de l'itinéraire initiatique mais également $d u$ voyage des initiés. Cette embarcationmonstre est évidemment le symbole du rapport entre intérieur et extérieur, c'est-àdire entre soi et dépassement de soi dans une communauté de croyances.

Bateau des dieux Scandinaves (Skidbladnir, Muspell, Ringhorn), barge royale des rois siamois aux proues en forme de naga ou de garuda ${ }^{5}$, barque birmane royales ${ }^{6}$, formes symbolisant les animaux mythiques (galon, karawek, hansasa, udon, kinari, kinara, naga), barques de Patani ${ }^{7}$, bateaux moken qui symbolisent les paroles d'une reine mythique, bateaux des saints de la chrétienté..., le bateau est bien l'instrument technique essentiel du support des croyances.

\section{Un ordre sociopolitique : de la pirogue monoxyle au bateau de planches; du nomade au sédentaire}

l'influence de la Chine que l'on a trop tendance à négliger.

«On y rencontre (îles Banda, Moluques) une étrange pirogue où il semble qu'un dragon fantastique ait pris place. C'est probablement l'extrême limite sud-est de l'influence culturelle chinoise qui a fait embarquer ce monstre sur ces pirogues malaises; les bateaux dragons de Chine et les pirogues monstrueuses des Cambodgiens sont identifiés à l'animal lui-même ; l'avant de la pirogue est une tête de monstre et l'arrière sa queue ; ses pattes traînent dans l'eau de chaque côté du corps ; à Banda, au contraire, la pirogue lance un rode protecteur en avant et en arrière du dragon qui semble ainsi confortablement installé à bord.» (Poujade 1946 : 284-285).

Tous les thèmes symboliques, rituels et religieux sont liés : bateau, maison, oiseau, arbre, serpent (les symboles lunaire et solaire paraissent trop généraux pour être pris en considération ici); les Batak de Sumatra, les Toraja des Célèbes ou les Nagju de Bornéo sont les meilleurs représentants de l'art symbolique et rituel reliant ces éléments mythologiques. Un des thèmes dominants de la culture ngaju représente un oiseau, un arbre et une construction posée sur la poupe d'un bateau. On trouve aussi deux oiseaux, représentant les divinités du monde supérieur et inférieur, une symbolique qui s'avère religieuse avant tout, l'oiseau étant l'animal psychopompe et l'abri, la maison, le temple posé sur un bateau étant le symbole bouddhiste du voyage à travers les renaissances et les flots tumultueux de la vie. D'ailleurs, nous trouvons des «bateaux des morts » un peu partout parmi ces populations, ornés d'un oiseau (calao en Asie du Sud-Est insulaire, tourterelle en Asie du Sud-Est continentale) ${ }^{8}$. Nous avons vu en outre que l'oiseau apparaît techniquement et symboliquement lié aux bateaux asiatiques et que le vocabulaire de la maison peut-être employé pour la technologie navale; notons enfin que l'arbre, centre du monde, Nombril du Monde diraient les Malais, peut devenir un bateau et qu'il dénote l'épreuve, le courage, le mystère, le 
passage. On retrouve donc au centre du monde un arbre gigantesque, axe de l'univers, que surplombe un oiseau (souvent un garuda dans les populations influencées par le bouddhisme ou des divinités des mondes supérieur et inférieur). À son sommet, se trouve le nid du volatile ; et il s'élève au-dessus d'une mer tourmentée, qu'infestent des monstres marins, lesquels dévorent ceux qui ont commis des péchés. Mais c'est aussi le lieu des renaissances, tous les thèmes traditionnels, bouddhistes et même musulmans se retrouvant dans cet arbre cosmique aux significations multiples.

31 À Halmahera (cf. C. Barraud, citée par P.-Y. Manguin), à la suite de la division entre terre et mer associée à une différence entre féminin et masculin, nous retrouvons une symbolique binaire ( $c f$. L. Gansser et J. Ivanoff), où le gouvernail et d'autres pièces sont assimilés à des objets sexuels (V. Arnaud) et sexués, et le bateau est lui-même féminin (le patia, fille des charpentiers, cf. L. Varadarajan). Le bateau est aussi le lieu de l'union, de la genèse d'une hiérarchie (chez les Malais) ou à l'inverse celui d'une absence de hiérarchie (chez les Moken). Le bateau pénètre l'eau du fleuve lors des régates cambodgiennes comme les génies ophidiens pénètrent dans le champ pour le féconder ; sa construction est assimilée à une naissance, on lui bouche le nombril (dans les Célèbes); il existe donc une connotation sexuelle très forte liant le bateau à la fécondité (voir le rôle de la femme enceinte dans les rituels de lancement, le symbolisme sexuel prononcé en Insulinde où les différentes parties du bateau sont sexuées, et même à Tahiti où une activité féconde n'est permise que par la présence de constituants de sexe opposé). Mentionnons enfin, à propos de ces rituels de fécondité, les offrandes et parfois les sacrifices, autrefois peut-être humains (H. Guiot, C. Coiffier). De ce fait, le bateau introduit symboliquement un référent social qui puise ses racines dans le mythe. C'est sa force; il révèle l'âme profonde d'un peuple en lui constituant l'objet d'un culte social.

Le monoxyle, double de l'homme et rappel de l'unité mythique des éléments, permet à l'humanité de ne jamais perdre le chemin qui unit l'homme à l'univers. À l'opposé, les bateaux en planches ont privé l'homme de ce contact primordial en standardisant la fabrication et les rituels. En séparant le corps humain de son expression technique inscrite dans le monoxyle, le bateau en planches va devenir le pivot d'une identité sociale, le symbole d'une hiérarchie. Le technique et le symbolique se développent donc dans un même mouvement.

\section{BIBLIOGRAPHIE}

Bachelard, Gaston, 1976, L'eau et les rêves. Paris : Corti.

Braginsky, Vladimir I, 1988, «A preliminary reconstruction of the rencong version of "Poem of the Boat" », BEFEO (Bulletin de l'Ecole Française d'Extrême-Orient) 77 : 263-301.

- 1998, "Sufi boat symbolism : problem of origin and evolution », Indonesia and the Malay World 26 (74) : 50-64. 
Charney Michael W., 1997, « Shallow-draft Boats, Guns, and the Aye-ra-wa-ti. Continuity and Change in Ship Structure and River Warfare In Precolonial Myanma », Oriens Extremus 40, 1 : 16-63.

Christinger R., P. Solier et F. Siegenthaler, 1980, Récits mythiques et symbolisme de la navigation. Paris : Dervy-Livres (« L'œuvre secrète »).

Corbin, Alain, 1988, Le Territoire du vide. L'occident et le désir du rivage 1750-1840. Paris : Flammarion («Champs»).

Ivanoff, Jacques, 1999, The Moken boat : Symbolic technology. Bangkok : White Lotus.

Hornell, James, 1918, « The origins and Ethnological Significance of Indian Boats Designs », Memoirs XI of The Asiatic Society of Bengal VIII, 3.

Jung, Carl G, 1964, Métamorphoses et symboles de la libido. Paris : Gallimard (« Idées ») (1ère édition 1933).

1932, Dialectique du moi et de l'inconscient. Paris : Montaigne.

Neyret, Jean S. M., 1974, Pirogues océaniennes. Tome I : Mélanésie, Tome II : Polynésie, Micronésie, Indonésie, Inde, Autres continents. Paris : Association des amis du musée de la marine.

Pâris, Edmond (Amiral), 1841, Essai sur la construction navale des Peuples extra-européens ou Collection des navires et pirogues construites par les habitants de l'Asie, de la Malaisie, du Grand Océan et de l'Amérique. Paris.

Planhol (de), Xavier, 2000, L'Islam et la mer. La mosquée et le matelot. VII ${ }^{e}-X X^{e}$ siècles. Paris : Perrin (« Histoire et décadences »).

Poujade, Jean, 1946, La route des Indes et ses navires. Paris : Payot.

\section{NOTES}

1. On retrouve évidemment ce symbole dans le monde chrétien: "L'église revêt la figure du bateau, le Saint-Esprit, celle du gouvernail qui conduit au havre éternel, objet de désir du chrétien ; alors que le péché fait dériver, loin de la route du salut. » (Corbin 1988 :19). La mer, démoniaque pour l'église, et qui abrite les monstres, justifie ainsi les exorcismes et laisse la place aux croyances populaires.

2. Notons ici les problèmes auxquels se heurtent les musulmans qui meurent en mer et qui devraient en théorie être mis en terre. C'est une des raisons pour lesquelles nous avons observé que les musulmans vivant sur certaines îles, à la frontière birmano-thaie partent en mer pour chercher les cadavres et les enterrer (à propos de la mort en mer, cf. de Planhol 2000 : 466).

3. Remarquons à ce propos que la mer, si décriée parfois par les chrétiens et les musulmans, a permis le développement de ces religions en favorisant le voyage des missionnaires.

4. Tout en s'appropriant «l'esprit » de la technique traditionnelle, le monde moderne le détruit en le vidant de sa substance rituelle, sociale et symbolique (bateaux de plaisance à propulsion « traditionnelle ", « liberté » assimilée à un nomadisme idéalisé...).

5. Khrut Tret Traichak, "garuda traversant les Trois Royaumes»; Narai Song Suban, "Vishnu chevauchant le garuda »; Anantakkharat, «Le grand roi naga à sept têtes »; Anekchatphuchong, «Les incarnations multiples du naga »; Suphannahong, « L'oie d'or », etc.

6. Let Ywe Gyi Hlaw Ka Taw Gyi, bateau de cérémonie, de course et de guerre, Nawa Rupa, le bateau des "neuf beautés»; Ma Noke Thida, dont la proue porte la figure d'un animal fabuleux, possédant un visage humain mais un double corps de lion, etc. 
7. Les kolè' décorés de naga, gago suro ou hastinliga.

8. Méfions-nous cependant du «tout rituel » car, comme le remarque judicieucement M. JacqHergoualc'h, les motifs des tambours de l'époque dongsonnienne qui associent fort à propos pour notre ouvrage l'oiseau et le bateau ne sont pas forcément des motifs religieux liés au chamanisme.

\section{RÉSUMÉS}

«The tree is of extreme height/Although enormous, it has a seed as its origin/Its perfect fruits are not hidden/Truly perfect, they are not veiled/Your boat is named Herald/The expert artisan named beauty/Has built [this] boat in the Estuary of the Omnipotent... (st. 8)/It is omnipotent, hard is the work/To go patiently around the world/And in various ways to return to the Sea of the Veiling One... (st. 58)/The Shore of Allah is the name of its [the boat's] anchorage.../The Centre of the Circles is the name of its sailing master.../His place is not behind the passengers, he knows when the wind changes.

This master is expert and wise/Navigating in the boundless sea... (sts. 79, 49-50)/They are birds, who climb mountains/These birds do not fly... (sts. 85-86)/Many of your ennemies will assail/Thy ship, if it comes upon reefs.../[They are all around] numerous reefs, distant shallows/ Treacherous rocks and islands (sts. 71, 76). ( (cite par V. Braginsky 1998 : 57-59). 